\title{
Fiber bundle model with stick-slip dynamics
}

\author{
Zoltán Halász and Ferenc Kun* \\ Department of Theoretical Physics, University of Debrecen, P.O. Box 5, H-4010 Debrecen, Hungary
}

(Received 20 May 2009; published 27 August 2009)

\begin{abstract}
We propose a generic model to describe the mechanical response and failure of systems which undergo a series of stick-slip events when subjected to an external load. We model the system as a bundle of fibers, where single fibers can gradually increase their relaxed length with a stick-slip mechanism activated by the increasing load. We determine the constitutive equation of the system and show by analytical calculations that on the macroscale a plastic response emerges followed by a hardening or softening regime. Releasing the load, an irreversible permanent deformation occurs which depends on the properties of sliding events. For quenched and annealed disorder of the failure thresholds the same qualitative behavior is found, however, in the annealed case the plastic regime is more pronounced.
\end{abstract}

\section{DOI: 10.1103/PhysRevE.80.027102}

There is a large variety of systems which undergo conformational changes when subjected to external mechanical loads. The dynamics of spatial rearrangements can usually be described by the stick-slip mechanism, i.e., when the local load exceeds some threshold value, subunits of the system increase their length leading to relaxation. The stored length which can be activated by external loading typically arises due to the existence of frictional contacts or chemical bonding between subunits. Several examples of such systems can be mentioned from the molecular scale organization of spider silk [1], through the chains of magnetic beads in magnetorheological fluids on the mesoscale [2,3], to the wire nets used to protect roads from rockfalls in mountains [4] on the macroscale. Experiments have revealed that the surprisingly high fracture toughness of spider silk is partly caused by the presence of blobs of protein molecules with a folded hairpin structure, which get unfolded under external loading [1]. A similar mechanical response has also been observed for biological tissues composed of interconnected bundles of fibrils [5]. In magnetorheological fluids particles of permanent magnetic moment aggregate and form chains aligned with the external field, which then modify the rheological properties of the fluid. Stretching and bending chains of particles of micrometer size by optical tweezers revealed that the anisotropic magnetic interaction and the frictional contact of particles result in a noisy response, i.e., particles undergo subsequent rearrangements increasing the length of the chain and reducing the reactant force [2,3]. A similar mechanism is exploited on the macroscale in wire nets, which cover steep walls in mountains protecting roads from rockfalls. In order to dissipate the kinetic energy of falling boulders, the wires form rings which can slide and collapse without breaking resulting in a large dissipation but keeping the integrity of the net [4].

In the Brief Report we present a micromechanical model which captures the main ingredients of the mechanical response of system which undergo conformational changes with stick-slip mechanism. Our model construction is based on fiber bundle models [6-11] extended in such a way that fibers undergo sliding events which gradually increase their

\footnotetext{
*feri@dtp.atomki.hu
}

PACS number(s): 46.50.+a, 62.20.M-, 81.40.Np

relaxed length when the local load exceeds some threshold values. After a large number of sliding events, fibers may also fail under a large enough external loads. We derive the constitutive equation of the system and show analytically that the sliding mechanism leads to macroscopic plasticity of the bundle and permanent deformation remains after the load has been released. We explore the case of both quenched and annealed disorder of sliding thresholds.

Our model consists of $N$ parallel fibers which have identical elastic properties characterized by the Young modulus $E$ [6-11]. Under an increasing external load $\sigma$ parallel to the fibers' direction, the fibers exhibit a linearly elastic behavior until the local deformation $\varepsilon_{i}$ reaches a threshold value $\varepsilon_{t h}^{i}$, $i=1, \ldots, N$. The key element of the model is that at the threshold the fiber does not break, instead it suffers sliding, i.e., its relaxed length increases until the fiber becomes capable to sustain the remaining load. The sliding threshold of fibers $\varepsilon_{t h}$ is a random variable with a probability density $p\left(\varepsilon_{t h}\right)$ and a distribution function $P\left(\varepsilon_{t h}\right)$ defined over the domain $\left[\varepsilon_{t h}^{\min }, \varepsilon_{t h}^{\max }\right]$. The sliding event is instantaneous, i.e., it does not take time. After the relaxation the fiber can be loaded again which may lead to further slidings. The total number of allowed sliding events (steps of extension) $k_{\max }$ is an important parameter of the model. When the fibers can slide more than ones $k_{\max }>1$, they either keep the initial sliding threshold for all slidings (quenched disorder), or can obtain a new threshold value each time from the same probability distribution (annealed disorder). The constitutive behavior of single fibers is illustrated by Fig. 1 for both types of disorder.

The sliding of a fiber means that its equilibrium length gets suddenly increased, which has the consequence that the fiber can only keep a lower load $\sigma_{i}=E\left(\varepsilon-\varepsilon_{t h}^{i}\right)$, where $\sigma_{i}$ denotes the local load of fiber $i, \varepsilon_{t h}^{i}$ is its sliding thresholds and $\varepsilon$ is the macroscopic strain of the system. Under a fixed external load $\sigma$, the other fibers of the bundle have to overtake the load dropped by the one which has just been extended. For the load redistribution we assume an infinite range of interaction, i.e., load sharing $[8,11]$. However, it does not imply that the load is everywhere the same in the bundle. At a given macroscopic deformation $\varepsilon$ the fibers have suffered a different number $k_{i}$ of slidings occurring at different threshold values, hence the load they keep will be 

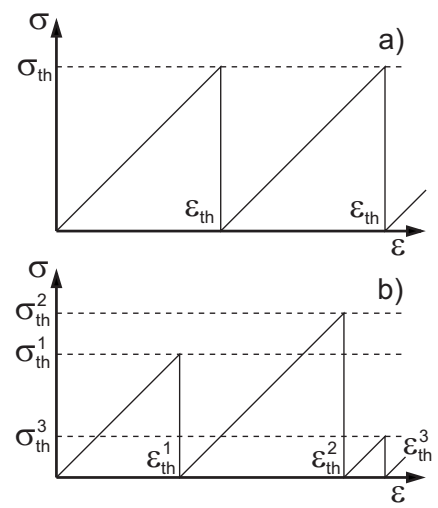

FIG. 1. Response of a single fiber for quenched (a) and annealed (b) disorder. When the failure threshold is reached the load on the fiber drops down to zero. The threshold can be constant (a) or new ones can be drawn from the same distribution (b).

different: $\sigma_{i}=E\left(\varepsilon-k_{i} \varepsilon_{t h}^{i}\right)$ for quenched, and $\sigma_{i}=E\left(\varepsilon-\varepsilon_{t h}^{i, 1}\right.$ $\left.-\varepsilon_{t h}^{i, 2}-\cdots-\varepsilon_{t h}^{i, k_{i}}\right)$ for annealed disorder $(i=1, \ldots, N)$, respectively. (A fuse model with a slightly similar microscopic dynamics was considered in Ref. [12], where fuses burn out due to gradual overheating.)

We start the analysis with the case of quenched disorder and assume that only a single sliding is allowed for the fibers $k_{\max }=1$. At the macroscopic deformation $\varepsilon$ the fibers with sliding thresholds $\varepsilon_{t h}^{i} \geq \varepsilon$ are still intact and have the local load $E \varepsilon$. However, those fibers which have already suffered a sliding $\varepsilon_{t h}^{i}<\varepsilon$ keep only the load $E\left(\varepsilon-\varepsilon_{t h}^{i}\right)$ so that the macroscopic constitutive equation follows as

$$
\sigma(\varepsilon)=E \varepsilon[1-P(E \varepsilon)]+\int_{\varepsilon_{t h}^{\min }}^{\varepsilon} p\left(E \varepsilon_{1}\right) E\left(\varepsilon-\varepsilon_{1}\right) d \varepsilon_{1} .
$$

Note that the integral in the second term is performed over the entire loading history of the system. In the integrand the probability density $p$ of the thresholds occurs since the load of the extended fibers depends on the precise value of the deformation where the sliding initiated. Allowing for two sliding events $k_{\max }=2$, at the macroscopic deformation $\varepsilon$ those fibers slided exactly once whose sliding threshold falls in the range $\varepsilon / 2<\varepsilon_{t h}^{i}<\varepsilon$, while the ones with $\varepsilon_{t h}^{i}<\varepsilon / 2$ already suffered two slidings, hence, $\sigma(\varepsilon)$ can be written as

$$
\begin{aligned}
\sigma(\varepsilon)= & E \varepsilon[1-P(E \varepsilon)]+\int_{\varepsilon / 2}^{\varepsilon} p\left(E \varepsilon_{1}\right) E\left(\varepsilon-\varepsilon_{1}\right) d \varepsilon_{1} \\
& +\int_{\varepsilon_{\text {th }}}^{\varepsilon / 2} p\left(E \varepsilon_{1}\right) E\left(\varepsilon-2 \varepsilon_{1}\right) d \varepsilon_{1} .
\end{aligned}
$$

For any arbitrary value of $k_{\max }$ the sliding threshold of fibers which are intact or suffered a $k$ number of sliding events 1 $\leq k \leq k_{\max }$ fall in the following subintervals of $\left[\varepsilon_{t h}^{\min }, \varepsilon_{t h}^{\max }\right]$

$$
\varepsilon<\varepsilon_{t h}^{i}, \quad \text { intact }
$$

$$
\frac{\varepsilon}{k+1}<\varepsilon_{t h}^{i}<\frac{\varepsilon}{k} \quad \text { sliding } \mathrm{k} \text { times, where } k<k_{\max }
$$

$$
0<\varepsilon_{t h}^{i}<\frac{\varepsilon}{k_{\max }} \text { sliding } k_{\max } \text { times. }
$$

The general form of the macroscopic constitutive equation can be obtained by integrating the load kept by the above subsets of fibers

$$
\begin{aligned}
\sigma(\varepsilon)= & E \varepsilon[1-P(E \varepsilon)]+\sum_{k=1}^{k_{\max }-1} \int_{\varepsilon /(k+1)}^{\varepsilon / k} p\left(E \varepsilon_{1}\right) E\left(\varepsilon-k \varepsilon_{1}\right) d \varepsilon_{1} \\
& +\int_{\varepsilon_{t h}^{\min }}^{\varepsilon / k_{\max }} p\left(E \varepsilon_{1}\right) E\left(\varepsilon-k_{\max } \varepsilon_{1}\right) d \varepsilon_{1} .
\end{aligned}
$$

Note that in Eq. (4) the fibers retain their initial stiffness after suffering $k_{\max }$ sliding events, which is expressed by the last term. It can be seen that for small deformations $\varepsilon \rightarrow 0$ only the intact fibers are relevant which result in a macroscopic linear behavior with a Young modulus equal to that of the individual fibers. For very large deformations $\varepsilon \rightarrow \infty$, practically all fibers have suffered $k_{\max }$ restructuring events so that only the last term of Eq. (4) survives which can be further written as

$$
\sigma(\varepsilon) \sim E \varepsilon-k_{\max } E \int_{\varepsilon_{\text {th }}^{\min }}^{\varepsilon / k_{\max }} p\left(\varepsilon_{1}\right) \varepsilon_{1} d \varepsilon_{1} .
$$

The integral in the second term converges to the average value of the thresholds $\left\langle\varepsilon_{t h}\right\rangle$, hence, we obtain $\sigma(\varepsilon) \sim E \varepsilon$ $-k_{\max } E\left\langle\varepsilon_{t h}\right\rangle$. The result implies that the bundle has an asymptotic linear behavior with the initial value of the Young modulus. When unloading the system $\sigma(\varepsilon) \rightarrow 0$, the fibers simply relax with a linearly elastic response since there is not any mechanism in the model to decrease the equilibrium length of fibers. It has the consequence that as the load is released, the system relaxes along a straight line of slope $E$ equal to the Young modulus of fibers and an irreversible remaining deformation occurs $\varepsilon_{r}$, whose maximum value $\varepsilon_{r}^{\max }$ is proportional to the average slip length $\left\langle\varepsilon_{t h}\right\rangle$ and the number of sliding events $k_{\max }$ allowed $\varepsilon_{r}^{\max }=k_{\max }\left\langle\varepsilon_{t h}\right\rangle$. Unloading the bundle after reaching a macroscopic deformation $\varepsilon_{m}$ the remaining irreversible deformation $\varepsilon_{r}$ can be obtained as $\varepsilon_{r}=\varepsilon_{m}-\sigma\left(\varepsilon_{m}\right) / E$. For the purpose of explicit calculations we used a Weibull distribution for the sliding thresholds with the cumulative distribution $P\left(\varepsilon_{t h}\right)=1-e^{-\left(\varepsilon_{t h} / \lambda\right)^{m}}$ over the range $0 \leq \varepsilon_{t h}<+\infty$, where $\lambda$ sets the scale of the threshold values and $m$ denotes the Weibull exponent. In all the calculations $\lambda=1$ was chosen. The constitutive curve of the model is presented in Fig. 2 for the case of $m=1$, i.e., exponential distribution. It can be observed that increasing $k_{\max }$ the asymptotic linear part of $\sigma(\varepsilon)$ is preceded by a longer and longer plateau regime indicating a plastic response of the system. Figure 3 presents the remaining permanent deformation $\varepsilon_{r}$ of the bundle measured after unloading the system from the maximum deformation $\varepsilon_{m}$ of the loading process. One can observe that $\varepsilon_{r}$ tends to the maximum value $k_{\max }\left\langle\varepsilon_{t h}\right\rangle$ as $\varepsilon_{m}$ increases.

In realistic situations it is typical that after a certain number of sliding events the relaxed length of the subunits of the system cannot be further extended, which then results in 


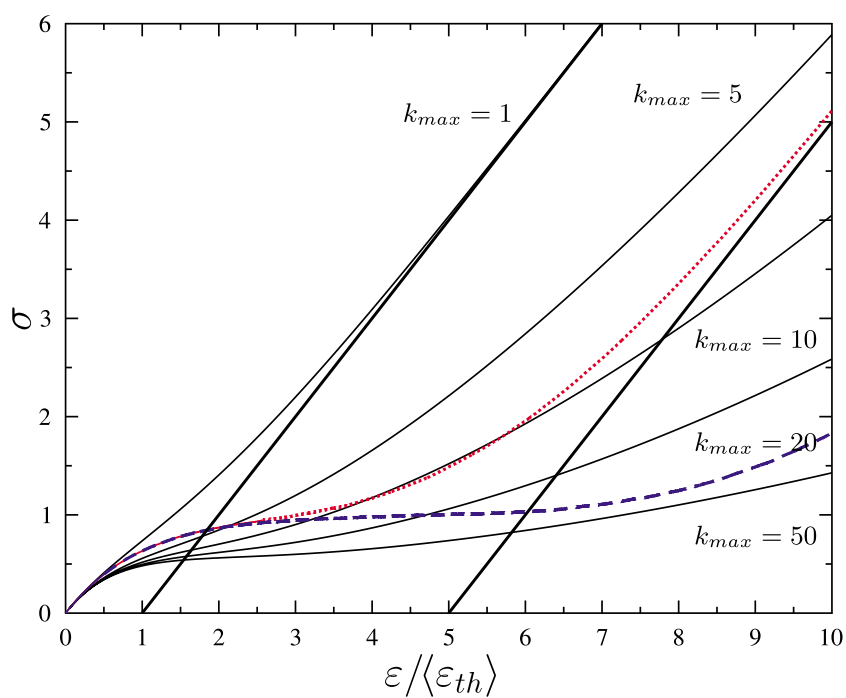

FIG. 2. (Color online) Constitutive behavior of the bundle with exponentially distributed quenched failure thresholds. $\sigma(\varepsilon)$ tends to an asymptotic linear response preceded by a more and more horizontal plateau when $k_{\max }$ increases. The intersection of the asymptotic straight lines with the horizontal axis indicates the value of $\varepsilon_{r}^{\max }$ obtained when unloading the system. For annealed disorder the cases of $k_{\max }=5$ (red, dotted line) and 10 (blue, dashed line) are shown.

breaking. Such cases can be captured by the model with the assumption that after a fiber has slided $k_{\max }$ times it breaks irreversibly, i.e., its stiffness is set to zero. The constitutive equation of the sliding-breaking system can be obtained analytically by skipping the last term of Eq. (4) which has the consequence that for large deformations $\varepsilon \rightarrow \infty$ the stress tends to zero $\sigma(\varepsilon) \rightarrow 0$ indicating that the bundle gradually looses its load bearing capacity. Figure 4 presents a comparison of constitutive curves Eq. (4) with hardening and failure after $k_{\max }$ slidings. It is important to emphasize that due to the breaking of fibers, the unloading Young modulus of the

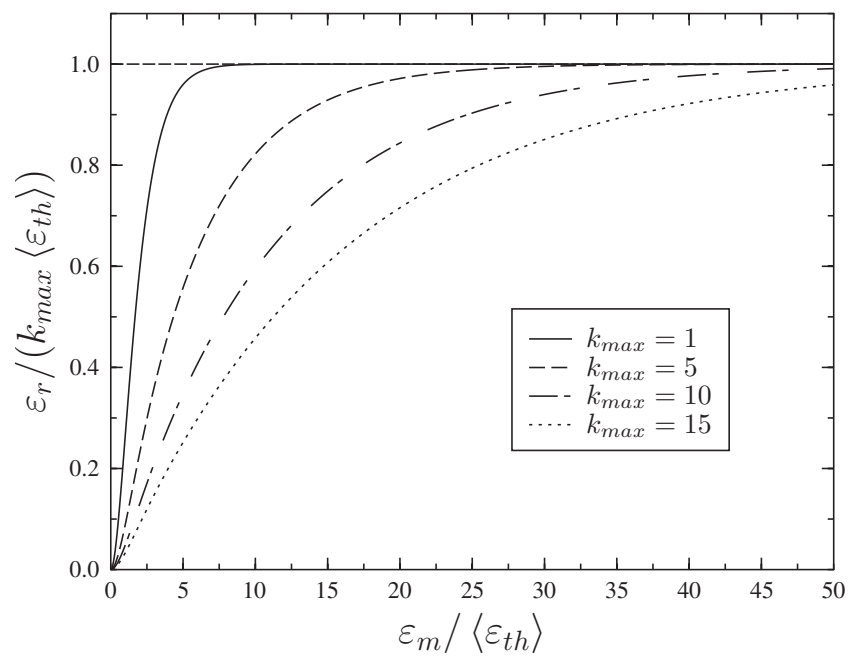

FIG. 3. Remaining deformation $\varepsilon_{r}$ as a function of the maximum deformation $\varepsilon_{m}$ reached before the unloading starts for different values of $k_{\max } . \varepsilon_{r}$ and $\varepsilon_{m}$ are normalized by the average sliding threshold $\left\langle\varepsilon_{t h}\right\rangle$ and the maximum value $\varepsilon_{r}^{\max }$, respectively.

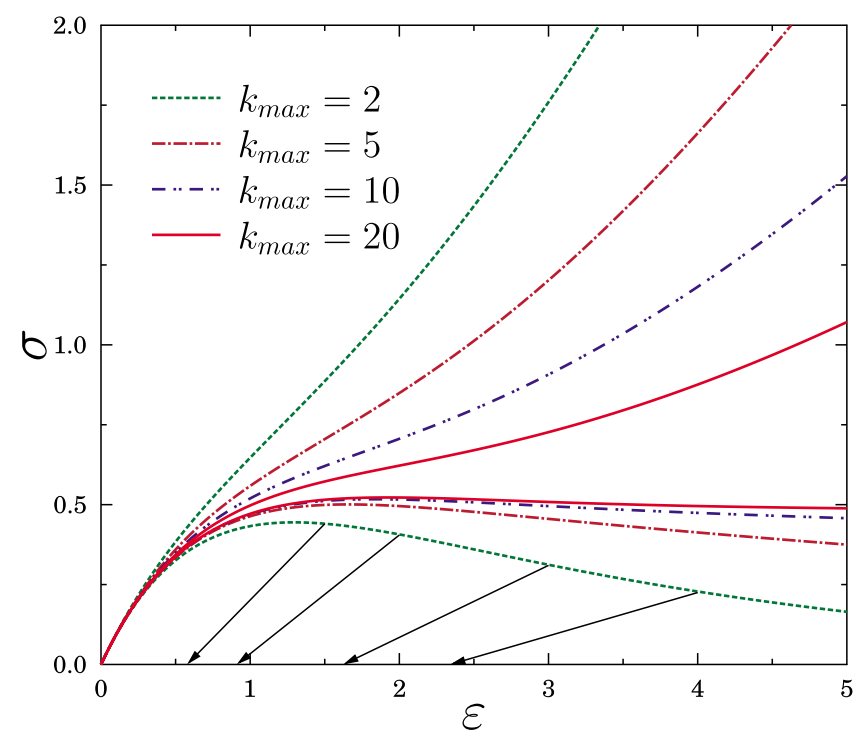

FIG. 4. (Color online) Comparison of the constitutive curves of the system with hardening (no failure) and failure after $k_{\max }$ sliding events. The arrows represent unloading curves for $k_{\max }=2$ with a decreasing Young modulus.

system is a decreasing function of the maximum deformation $\varepsilon_{m}$ achieved before the load is released so that the remaining deformation $\varepsilon_{r}$ takes the form $\varepsilon_{r}=\varepsilon_{m}-\sigma\left(\varepsilon_{m}\right) / E[1$ $\left.-P\left(E \varepsilon_{m} / k_{\text {max }}\right)\right]$. In Fig. 4 the arrows indicate unloading curves where the decreasing unloading modulus can also be observed. Under stress controlled loading the constitutive curve $\sigma(\varepsilon)$ can only be realized up to the maximum, where macroscopic failure occurs. The value of the maximum defines the fracture strength $\sigma_{c}=\sigma\left(\varepsilon_{c}\right)$ of the sliding-breaking bundle, where the critical deformation $\varepsilon_{c}$ can be determined numerically from the equation $1=\varepsilon_{c}\left[\sum_{k=1}^{k_{\max }-1}(1 / k) p\left(E \boldsymbol{\varepsilon}_{c} / k\right)\right.$ $\left.-\left(k_{\max }-1\right) / k_{\max } p\left(E \varepsilon_{c} / k_{\max }\right)\right]$. Numerical analysis of the above equation shows that both $\varepsilon_{c}$ and $\sigma_{c}$ are increasing functions of $k_{\max }$ converging to finite values.

In the case of annealed disorder, the fibers get a new threshold value from the same probability distribution after each sliding event [see also Fig. 1(b)]. The new threshold may represent that the physical properties of the new conformation attained by the fiber are different from the previous ones including also the effect of thermal noise. The macroscopic load $\sigma$ on the system can be obtained by summing the load above subsets of fibers with different sliding numbers $k=0,1, \ldots, k_{\text {max }}$ as $\sigma(\varepsilon)=\sigma_{0}(\varepsilon)+\sigma_{1}(\varepsilon)+\cdots+\sigma_{k_{\max }}(\varepsilon)$. Due to the independence of subsequent thresholds the terms of the above constitutive equation can be cast in the forms $\sigma_{0}(\varepsilon)$ $=E \varepsilon[1-P(E \varepsilon)]$,

$$
\begin{aligned}
\sigma_{1}(\varepsilon)= & \int_{\varepsilon_{t h}^{\min }}^{\varepsilon} p\left(\varepsilon_{1}\right) d\left(\varepsilon_{1}\right) E\left(\varepsilon-\varepsilon_{1}\right)\left[1-P\left(\varepsilon-\varepsilon_{1}\right)\right], \\
\sigma_{2}(\varepsilon)= & \int_{\varepsilon_{t h}^{\min }}^{\varepsilon} \int_{\varepsilon_{t h}^{\min }}^{\varepsilon-\varepsilon_{1}} d \varepsilon_{1} d \varepsilon_{2} p\left(\varepsilon_{1}\right) p\left(\varepsilon_{2}\right) \\
& \times\left[1-P\left(\varepsilon-\varepsilon_{1}-\varepsilon_{2}\right)\right] E\left(\varepsilon-\varepsilon_{1}-\varepsilon_{2}\right) .
\end{aligned}
$$

The general case of arbitrary $k<k_{\text {max }}$ reads as 


$$
\begin{aligned}
\sigma_{k}(\varepsilon)= & \int_{\varepsilon_{t h}^{\min }}^{\varepsilon} \int_{\varepsilon_{t h}^{\min }}^{\varepsilon-\varepsilon_{1}} \cdots \int_{\varepsilon_{t h}^{\min }}^{\varepsilon-\varepsilon_{1}-\varepsilon_{2} \cdots-\varepsilon_{k}} \prod_{l=1}^{k} d \varepsilon_{l} p\left(\varepsilon_{l}\right) \\
& \times\left[1-P\left(\varepsilon-\sum_{l=1}^{k} \varepsilon_{l}\right)\right] E\left(\varepsilon-\sum_{l=1}^{k} \varepsilon_{l}\right),
\end{aligned}
$$

Finally, for $k=k_{\max }$ we get

$$
\begin{aligned}
\sigma_{k_{\max }}(\varepsilon)= & \int_{\varepsilon_{t h}^{\min }}^{\varepsilon} \ldots \int_{\varepsilon_{t h}^{\min }}^{\varepsilon-\varepsilon_{1}-\cdots-\varepsilon_{k_{\max }}} \prod_{l=1}^{k_{\max }} d \varepsilon_{l} p\left(\varepsilon_{l}\right) \\
& \times E\left(\varepsilon-\sum_{l=1}^{k_{\max }} \varepsilon_{l}\right) .
\end{aligned}
$$

Figure 2 shows also examples of the constitutive curve $\sigma(\varepsilon)$ of the fiber bundle with annealed disorder. We obtain qualitatively the same behavior with the same asymptote as with quenched disorder, however, the plastic plateau becomes significantly longer. The result shows that the extension of the plastic regime is determined by $\varepsilon_{r}^{\max }$, however, the precise functional form of $\sigma(\varepsilon)$ is sensitive to the type of disorder.

We presented a micromechanical model for systems which can extend their length in a series of stick-slip events when subjected to an external load. The model is an extension of fiber bundle models in such a way that single fibers have stick-slip rheology characterized by quenched or annealed threshold values of the local strain. We showed analytically that varying its parameters the model provides a broad spectrum of constitutive behaviors: for a large number of sliding events a plastic regime develops which is then followed by hardening (no breaking) and softening (fiber breaking). Unloading the system a permanent deformation remains which is a monotonically increasing function of the maximum deformation. If the fibers break after $k_{\text {max }}$ sliding events, the unloading modulus goes to zero with increasing deformation. The constitutive curves provided by the model have qualitative agreement with the measured response of stick-slip systems [1,5], however, for a quantitative comparison further improvement is needed: allowing for an increase or decrease in the Young modulus of single fibers after local sliding events would make the model more realistic. Assembling bundles of fibers to form a chain (serial coupling of fiber bundles) is also a promising direction to reproduce the mechanical response of biological tissues. Work in this direction is in progress.

F.Kun acknowledges support from the Hungarian Academy of Sciences.
[1] F. Vollrath and D. Porter, Soft Matter 2, 377 (2006).

[2] E. M. Furst and A. P. Gast, Phys. Rev. Lett. 82, 4130 (1999).

[3] S. L. Biswal and A. P. Gast, Phys. Rev. E 68, 021402 (2003).

[4] W. Gerber and B. Haller, in Environmental and Safety Concerns in Underground Construction, edited by $\mathrm{H}$. Lee, $\mathrm{H}$. Yang, and S. Chung, Proceedings of the 1st Asian Rock Mechanics Symposium (A.A. Balkema, Rotterdam, the Netherlands, 1997), pp. 915-920.

[5] B. E. Layton and A. M. Sastry, Acta Biomater. 68, 612 (2006).

[6] S. Pradhan, A. Hansen, and P. C. Hemmer, Phys. Rev. Lett. 95, 125501 (2005).
[7] D. De Tommasi, G. Puglisi, and G. Saccomandi, Phys. Rev. Lett. 100, 085502 (2008).

[8] F. Kun, S. Zapperi, and H. J. Herrmann, Eur. Phys. J. B 17, 269 (2000).

[9] D.-H. Kim, B. J. Kim, and H. Jeong, Phys. Rev. Lett. 94, 025501 (2005).

[10] N. Yoshioka, F. Kun, and N. Ito, Phys. Rev. Lett. 101, 145502 (2008).

[11] R. C. Hidalgo, Y. Moreno, F. Kun, and H. J. Herrmann, Phys. Rev. E 65, 046148 (2002).

[12] D. Sornette and C. Vanneste, Phys. Rev. Lett. 68, 612 (1992). 